\title{
Equal Pay for Equal Screening: Impact of Patient and Provider Gender on Reimbursement for Screening Colonoscopy
}

\author{
Zoe Post $^{1} \cdot$ Joshua Melson ${ }^{1}$
}

Accepted: 25 May 2021 / Published online: 10 June 2021

(c) The Author(s), under exclusive licence to Springer Science+Business Media, LLC, part of Springer Nature 2021

The purpose of screening colonoscopy is to reduce the burden of colorectal cancer (CRC), one of the most common causes of cancer-related death in the world. Since interval CRCs are inversely proportional to adenoma detection rates (ADR), high-quality colonoscopy ultimately reduces CRC morbidity and mortality [1]. The field of colonoscopic screening for CRC has therefore developed and applied metrics to reflect the performance of high-quality colonoscopy including ADR, cecal intubation rate and withdrawal time. The ideal financial compensation model for screening would preferentially reimburse high-quality colonoscopies that would be reflective of higher rates of CRC prevention, as opposed to basing reimbursement solely on the quantity of colonoscopies.

Female physicians remain substantially underrepresented in the field of gastroenterology; in 2019 less than $20 \%$ of the active endoscopists were female as compared with $40 \%$ of family medicine physicians [2]. In terms of the distribution in gastroenterology fellowships, $34 \%$ of fellow trainees are female [3]. The salary gap between male and female gastroenterologists exceeds $20 \%$ [4, 5]. A more recent study demonstrated that the salary gap correlates with the gender composition of a practice, where the salary gap increases in practices comprised of a majority of male physicians [6].

In the past decade, studies investigating the gender pay gap in medicine have focused on physician factors, including female physicians prioritizing flexible work schedules over higher pay, preferring academic positions, and negotiating less aggressively for higher salaries as compared with men. Another driver of the gender-based pay gap can be the work

Joshua Melson

Joshua_Melson@rush.edu

Zoe Post

Zoe_Post@rush.edu

1 Division of Digestive Diseases, Rush University Medical Center, 1725 W Harrison St Suite 207, Chicago, IL 60612, USA distribution itself, where those having a higher procedure load based on volume and complexity of the procedures can expect greater financial compensation. The impact of this procedural work distribution in relation to the gender of the endoscopist on the gender-based pay disparity has not been well described.

In this issue of Digestive Diseases and Sciences, Andersson and co-authors performed a retrospective descriptive study which analyzed procedure time and gender differences in 16,573 screening colonoscopies performed over a three-year period in one large academic practice [7]. They hypothesized that the duration of a typical colonoscopy in a female patient is longer and that female endoscopists are more likely to perform colonoscopies on females than their male colleagues. Based on their findings, they explored the potential contribution of these differences to the gender pay gap in the physician Relative Value Unit (RVU)-based reimbursement model.

The key findings of the study in relation to screening colonoscopy were: (1) colonoscopy takes typically $8.8 \%$ longer to complete in female patients as compared with male patients; (2) female endoscopists perform an average of $71.2 \%$ of their colonoscopies on females as compared with male endoscopists, who perform an average of $50.8 \%$ on females; and (3) female patients had a lower ADR, which reduces the frequency of polypectomy and therefore decreases reimbursement per procedure. Taken together, longer colonoscopy procedure time and lower ADR decrease reimbursement for colonoscopies performed on female patients in an RVU-based model.

The authors should be commended for this novel study that attentively demonstrates how patient gender and patient's gender preferences of endoscopists interact and can ultimately impact reimbursement in a typical "productionbased" RVU model. Their study quantifies the disparity and how it can contribute to gender pay inequity.

The authors elucidate a specific systematic issue that can have impact on the gender-based reimbursement disparity 
among gastroenterologists. Based on the authors' calculations, including procedure time, percentage of female patients, and ADR, gender differences in screening colonoscopy may contribute to a gender pay gap close to $10 \%$. A reimbursement model for screening colonoscopy entirely dependent on procedural compensation as demonstrated in this study does not necessarily regard quality and, in addition, can lead to disparities in compensation for similar work based on patient selection.

Stepping back and considering the current prevalent RVU-based model, there are multiple alternate scenarios beyond gender that may impact reimbursement based on the specifics of the patient population in relation to the composition of the overall screening population. For instance, a practitioner who manages a high number of functional bowel disease patients would be expected to potentially generate lower RVUs per day of colonoscopy and therefore less compensation based on lower throughput due to the sedation challenges inherent in this population. Moreover, functional patients represent a population less likely to bear polyps due to the younger age at first colonoscopy with consequent reduced polypectomy RVU modifiers per procedure. A converse argument could be made for physicians who endoscope older patients, who likely bear a higher polyp burden than do younger patients, with consequent increase in the mean RVU modifiers.

Beyond the physician reimbursement concern, the current issue of physician-gender preference in gastroenterology patient-provider interactions is important. The authors discuss that patient preference for a gastroenterologist drives the gender proportional differences observed in their study. They reference a paper from 2002 that states that nearly half of female patients express a gender preference for a female endoscopist, whereas only 1 in 20 male patients expressed a gender preference [8]. Though the cause of this provider gender imbalance at their center is unclear, it may not solely be driven by patient preference but by other issues including endoscopist availability and scope of practice. Though highquality data describing recent trends of patient preference for a gender-specific endoscopist are limited, a recent study conducted in a primary care setting confirms the enduring preference of female patients for female endoscopists [9].

Gender is a factor that can be impactful on colonoscopy for CRC screening participation rates. In a Korean cohort, female gender was associated with delayed surveillance colonoscopy [10]. Female patients in an American cohort were more likely to no-show for a scheduled colonoscopy [11]. Currently, over 20 million eligible patients aged 50 years and over in the USA fail to undergo any appropriate CRC screening. If metrics could be developed that could efficiently identify the subset of patients who fail to undergo screening due to concerns about provider gender or other provider characteristics, this information could then potentially be used to modify the screening process for this subset of patients in order to align them with the provider who will increase the probability that screening will occur.

What steps can be taken to address the financial imbalance that female endoscopists can face based on their female patient's preferences that they perform their colonoscopies? The researchers propose that one possible approach could be to adjust RVUs for colonoscopies performed on females to compensate for the longer procedure times and lower polypectomy rates. Other subspecialties, including urology and gynecology, reimburse similar procedures done in males and females at different rates [12]. Prioritizing a financial compensation model governing colonoscopies that adjusts for providers managing female patients could have unpredicted and unwanted consequences such as adding unnecessary colonoscopies in women and disincentivizing male colonoscopies, while the latter group is at higher risk for developing CRC. Furthermore, if a gender-based reimbursement system is implemented, it would be unclear how it would be implemented, since personal identification and anatomical gender may be discordant.

Unfortunately, a large proportion of patients currently fails to undergo CRC screening. Reliance on opportunistic colonoscopy typically reaches a threshold of screening uptake that falls short of ideal [13]. It would therefore be interesting to investigate how an alternate screening model in which patients undergo colonoscopy only after a positive stool test impacts gender pay disparity in the current reimbursement model. Stool testing would select out a group with higher ADR in general and in turn perhaps reduce the disparity of RVUs based on gender while impacting a patient's request for a specific provider gender.

The Andersson et al. paper illustrates how the current reimbursement model is flawed and warrants re-evaluation, as it may reward providers based on relatively random criteria such as a greater frequency of male patients. Ideally, moving from a quantity-based model to a quality-based model that rewards high-quality colonoscopy based on established metrics such as ADR and on clear beneficial clinical outcomes such as interval colorectal cancer rates should not only positively address these disparities, but also improve the ultimate outcome, that is reducing CRC diagnoses and deaths.

\section{References}

1. Corley DA, Jensen CD, Marks AR et al. Adenoma detection rate and risk of colorectal cancer and death. $N$ Engl J Med. 2014;370:1298-1306.

2. Association of American Medical Colleges. 2020 Physician specialty data report: active physicians by sex and specialty, 2019, 
table 1.3. https://www.aamc.org/data-reports/workforce/inter active-data/active-physicians-sex-and-specialty-2019.

3. Association of American Medical Colleges. 2020 Physician specialty data report: ACGME Residents and fellows by sex and specialty, 2019, table 2.2. https://www.aamc.org/data-reports/inter active-data/acgme-residents-and-fellows-sex-and-specialty-2019.

4. Burke CA, Sastri SV, Jacobsen G et al. Gender disparity in the practice of gastroenterology: the first 5 years of a career. Am J Gastoenterol. 2005;100:259-264.

5. Singh A, Burke CA, Larive B, Sastri SV. Do gender disparities persist in gastroenterology after 10 years of practice? Am J Gastroenterol. 2008;103:1589-1595.

6. Whaley CM, Arnold DR, Gross N, et al. Practice composition and sex differences in physician income: observational study. $B M J$. 2020;370:2588.

7. Andersson KL, Ha JB, Abraczinskas DR, Campbell EJ, Richter JM. Gender differences in colonoscopy: implications for clinical practice and female gastroenterologists. Dig Dis Sci. (Epub ahead of print). https://doi.org/10.1007/s10620-021-07079-y.

8. Varadarajulu S, Petruff C, Ramsey WH. Patient preferences for gender of endoscopists. Gastrointest Endosc. 2002;56:170-173.

9. Fink M, Klein K, Sayers K et al. Objective data reveals gender preferences for patients' primary care physician. J Prim Care
Community Health. 2020. https://doi.org/10.1177/2150132720 967221.

10. Tae CH, Moon CM, Kim SE et al. Risk factors of nonadherence to colonoscopy surveillance after polypectomy and its impact on clinical outcomes: a KASID multicenter study. $J$ Gastroenterol. 2017;52:809-817.

11. Greenspan M, Chehl N, Shawron K et al. Patient non-adherence and cancellations are higher for screening colonoscopy compared with surveillance colonoscopy. Dig Dis Sci. 2015;60:2930-2936.

12. Benoit MR, Ma JF, Upperman BA. Comparison of 2015 Medicare relative value units for gender-specific procedures: gynecologic and gynecologic-oncologic versus urological CPT coding. Has time healed gender-worth? Gynecologic Oncology. 2017;144(2):336-342.

13. Melson JE, Imperiale TF, Itzkowitz SH et al. AGA white paper: roadmap for the future of colorectal cancer screening in the United States. Clin Gastroenterol Hepatol. 2020;18:2667-2678.e2.

Publisher's Note Springer Nature remains neutral with regard to jurisdictional claims in published maps and institutional affiliations. 